\title{
RIQUEZA Y DISTRIBUCIÓN DE HELECHOS Y LICÓFITOS EN el gradiente altitudinal del Cofre de Perote, CEntro de Veracruz, MÉxico
}

\author{
César I. Carvajal-Hernández ${ }^{1}$ y Thorsten Krömer \\ Centro de Investigaciones Tropicales, Universidad Veracruzana, Xalapa, Veracruz, México. \\ 1Autor para la correspondencia: cesar.carvajalh@gmail.com
}

\begin{abstract}
Resumen: Se presenta el listado de las especies de helechos y licófitos registrados a lo largo del gradiente altitudinal (20-3,500 m s.n.m.) del Cofre de Perote, Veracruz, abarcando ambientes contrastantes y bosques con diferentes grados de perturbación, así como vegetación azonal. Se establecieron 15 a 25 parcelas de $20 \times 20 \mathrm{~m}$ en ocho pisos altitudinales, separados por $500 \mathrm{~m}$ de altitud. En cada parcela, se registraron las especies terrestres y epífitas del sotobosque (hasta una altura aproximada de $8 \mathrm{~m}$ ). En 135 parcelas, se registraron 140 especies y cuatro variedades de helechos (22 familias, 59 géneros), así como 11 especies de licófitos (dos familias, tres géneros), representando el 27,5\% de la pteridoflora de Veracruz. Dos especies del género Elaphoglossum (E. pallidum, E. squarrosum) y Polystichum muricatum, registradas a 2,500 m, son nuevos registros para el estado. El número de especies es contrastante en diferentes pisos altitudinales del gradiente y en las elevaciones medias con bosque mesófilo de montaña entre 1,500 y 2,500 m se presentan los valores máximos de riqueza. En los extremos superior e inferior del gradiente, caracterizado por bosque de coníferas y selva mediana subcaducifolia respectivamente, el número de especies disminuye. Se destaca la riqueza del bosque mesófilo y la pérdida de especies del mismo cuando existe una perturbación de origen antrópico. Además, la vegetación azonal en cañadas y al lado de ríos representa un reservorio importante de la pteridoflora, lo que resalta su valor para la conservación.
\end{abstract}

Palabras clave: bosque de Pinus-Quercus, bosque mesófilo de montaña, inventario florístico, perturbación antrópica, pteridoflora.

\begin{abstract}
We present a list of ferns and lycophytes recorded along the elevational gradient (20-3,500 $\mathrm{m}$ a.s.1.) at the Cofre de Perote, Veracruz, including contrasting environments with different degrees of forest disturbance as well as azonal vegetation. We established 15-25 plots of $20 \times 20 \mathrm{~m}$ each at eight elevational steps separated by $500 \mathrm{~m}$ in elevation. In each plot we recorded all terrestrial and epiphytic understory species (up to a height of $8 \mathrm{~m}$ ). In the 135 plots, we found 140 species and four varieties of ferns (22 families, 58 genera) and 11 species of lycophytes (two families, three genera), representing $27.5 \%$ of the pteridophytes of Veracruz. Two species of the genus Elaphoglossum (E. pallidum, E. squarrosum) and Polystichum muricatum, found at 2,500 $\mathrm{m}$ are new state records. The number of species is contrasting at different elevational steps of the gradient and mid-elevations with humid montane forest between 1,500 and 2,500 m showed maximum richness values. At the upper and lower ends of the gradient, characterized by coniferous forest and deciduous forest, respectively, the number of species decreased. The richness of the humid montane forest and its loss of species caused by anthropogenic disturbance are noteworthy. Furthermore, the azonal vegetation at ravines and riversides represents an important reservoir for the pteridophyte flora, which highlights its conservation value.

Key words: anthropogenic disturbance, floristic inventory, humid montane forest, Pinus-Quercus forest, pteridophyte flora.
\end{abstract}

$\mathbf{L}$ a falta de conocimiento sobre las especies de plantas que habitan en determinadas regiones de México, es una necesidad urgente de atender (Magaña y Villaseñor, 2002). Veracruz, además de ser uno de los tres estados más biodiversos de México, es una de las entidades del país donde se han realizado grandes esfuerzos por conocer su flora, reflejados en los avances de la Flora de Veracruz (GómezPompa, 2010). Sin embargo estos trabajos aún distan mu- cho de ser concluyentes, debido a la falta de tratamientos y actualizaciones taxonómicas de diversas familias. Este es el caso de los helechos y licófitos, de los cuales solo se han publicado nueve de las 28 familias que están representadas en Veracruz (obs. pers.). Además una parte sustancial de esta información es desactualizada debido a que se publicaron en las décadas de 1980 y 1990.

Actualmente se estima que en Veracruz existen cerca de 
7,800 especies de plantas vasculares (Castillo-Campos et $a l ., 2011)$, de las cuales 564 especies corresponden a helechos y licófitos (Tejero-Díez et al., 2011; Krömer et al., 2015); esto representa el $55 \%$ de la pteridoflora de México y lo coloca en el tercer estado con mayor riqueza de especies de este grupo, solo después de Oaxaca y Chiapas (Mickel y Smith, 2004). Sin embargo, muchas de estas especies en Veracruz son consideradas como raras y amenazadas por factores antrópicos, ya que algunas sólo se registran de pocas y/o antiguas colectas y el conocimiento de su distribución es fragmentario (Tejero-Díez et al., 2011; Krömer et al., 2013a; Carvajal-Hernández et al., 2014; Acebey et al., 2015; Armenta-Montero et al., 2015).

La alta riqueza de especies registradas en Veracruz es producto de una gran heterogeneidad ambiental, originada por una topografía accidentada, un amplio intervalo altitudinal (desde el nivel del mar hasta más de $5,000 \mathrm{~m}$ ) y la confluencia de una amplia variedad de climas y suelos (Soto-Esparza y Geissert, 2011). Estas condiciones permiten la presencia de diferentes tipos de vegetación, desde los fríos y secos en zonas de alta montaña hasta los cálido-húmedos en zonas bajas.

Un gradiente altitudinal de Veracruz que permite observar dichos cambios en el clima y la vegetación es el que inicia en la llanura costera del centro del estado y termina en las partes altas del Cofre de Perote en dirección este. El contraste en la vegetación de este gradiente ha sido reconocido incluso desde Alexander von Humboldt en 1804, quien lo cita señalando lo siguiente: "En ninguna parte se deja ver mejor el admirable orden con que las diferentes asociaciones de vegetales van sucediéndose, unas arriba de las otras, que cuando uno va subiendo desde Veracruz hacia la meseta de Perote (...) en el espacio de pocas horas, recorre el hombre de ciencia toda la escala de vegetación" (von Humboldt, 1822; Zamudio y Butanda, 1999).

Dos siglos después, la vegetación natural a lo largo del gradiente del Cofre de Perote se encuentra reducida drásticamente y en la actualidad solo se encuentran fragmentos aislados de la vegetación original (Ellis et al., 2011). Esta situación ha sido motivo para que la Comisión Nacional para el Conocimiento y Uso de la Biodiversidad designara dos sitios prioritarios para la conservación en la zona, el primero corresponde a la parte alta de la cuenca del río La Antigua, la cual es una zona con bosque mesófilo de montaña de prioridad critica para su conservación, debido a las amenazas provocadas por las actividades antropogénicas (CONABIO, 2010). El segundo se encuentra en la zona baja del gradiente y forma parte de la cuenca del río Actopan, con fragmentos aislados de encinares tropicales, los cuales constituyen remanentes del Pleistoceno de origen boreal que sustituyeron la vegetación tropical durante las últimas glaciaciones (Arriaga et al., 2000).

Algunas zonas de este gradiente altitudinal han sido estudiadas desde diferentes aspectos botánicos y éstas cuen- tan con listados florísticos locales que incluyen especies de helechos y licófitos (p. ej. Hietz y Hietz-Seifert, 1995; Gómez-Díaz, 2010; Hernández-Rojas, 2010; Salazar-Rodríguez, 2010), aunque en su mayoría, se trata de tesis que no han sido publicadas. A pesar de esos esfuerzos particulares, no existen trabajos que aborden inventarios florísticos realizados a través de un muestreo sistemático que incluya un área tan amplia y heterogénea. Tampoco existe un listado completo y actualizado para estos importantes grupos, los cuales en conjunto conforman el segundo más diverso de las plantas vasculares (Kreft et al., 2010) y se sabe que alcanza su mayor riqueza y abundancia en regiones húmedo montañosas del Neotrópico (Moran et al., 1995; Kluge y Kessler, 2005; Watkins et al., 2006a; Kessler et al., 2011a; Salazar et al., 2015).

En México se han realizado pocos estudios que documenten la riqueza de la flora vascular o de grupos específicos en gradientes de altitud (Hietz y Hietz-Seifert, 1995; Vázquez y Givnish, 1998; Campos-Salas, 2011; Krömer et al., 2013b). Sin embargo, estos no se realizaron en gradientes completos (desde el nivel del mar hasta las zonas altas y frías de las montañas) y además solo la tesis de CamposSalas (2011) se centra en el estudio de helechos y licófitos. Ante ese vacío de conocimiento florístico, en el presente trabajo se incluye el listado de especies de estos grupos registradas a lo largo del gradiente altitudinal del Cofre de Perote, ubicado en el centro de Veracruz, abarcando ambientes y composición florística contrastantes a lo largo de las distintas elevaciones. Además se documentan las especies en vegetación con perturbación de origen antrópico en cada piso altitudinal estudiado. El objetivo de este trabajo es actualizar el conocimiento de la pteridoflora de Veracruz en una región fuertemente antropizada, pero de gran importancia ecológica, razones que la convierten en prioritaria para su conservación.

\section{Materiales y métodos}

Área de estudio. El gradiente altitudinal estudiado se encuentra en la región central del estado de Veracruz. El intervalo de altitud tiene como límite superior la ladera alta del Cofre de Perote en 3,500 m (19० 31' 10" N $-97^{\circ} 09^{\prime}$ $27^{\prime \prime} \mathrm{O}$ ), mientras que el extremo inferior se localiza en el nivel del mar, específicamente en el Centro de Investigaciones Costeras "La Mancha" (19³6' 02" N -96 22' 36 "O), perteneciente al Instituto de Ecología, A.C. (Figura 1). En la descripción geomorfológica de Veracruz, Geissert y Enríquez (2011) ubican a la zona como parte del Cinturón Neovolcánico Transversal y la caracterizan de la siguiente forma: "las montañas de laderas abruptas presentes en la parte alta del gradiente (superior a 1,400 m) están formadas por acumulaciones de andesita/basalto y flujos piroclásticos del Terciario; en el resto del transecto, se encuentran pequeños lomeríos formados por rocas volcánicas, paleogénicas a cuaternarias; en el extremo inferior se encuentra una plani- 


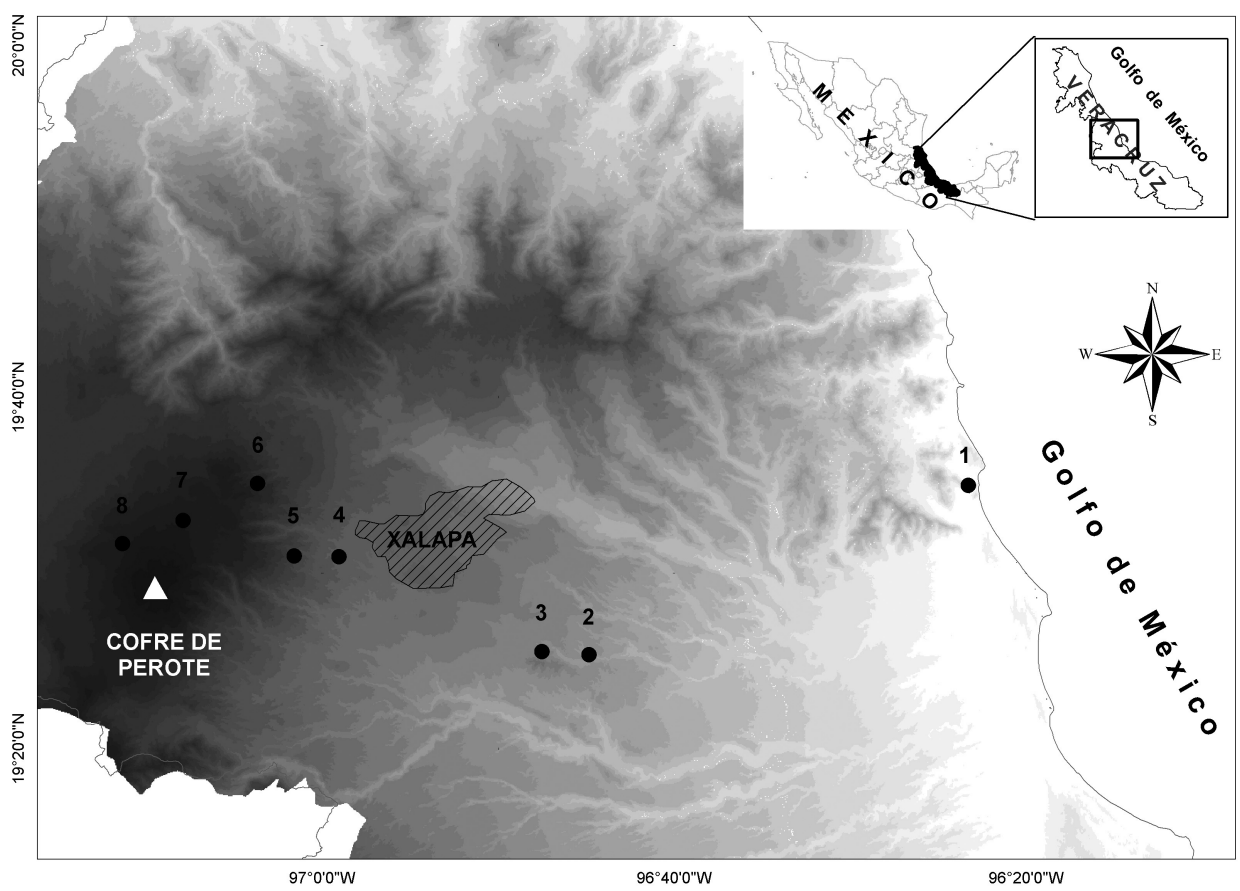

Figura. 1. Ubicación de los ocho sitios de estudio a lo largo del gradiente altitudinal en la vertiente del Golfo de México, centro del estado de Veracruz, México. Localidades de estudio: 1. La Mancha $(20 \mathrm{~m})$; 2. Palmarejo (500 m); 3. Chavarrillo (1,000 m); 4 . Los Capulines (1,500 m); 5. El Zapotal (2,000 m); 6. El Encinal (2,500 m); 7. Los Pescados (3,000 m); 8. El Conejo (3,500 m).

cie formada por acumulaciones fluviales y lagunas costeras como la de La Mancha".

A lo largo del gradiente de estudio las condiciones climáticas muestran una gama muy amplia, (Soto-Esparza y Giddings, 2011). La temperatura media anual tiene una disminución lineal con el aumento de la altitud, mientras que la precipitación anual es mayor en las partes medias y disminuye en los extremos del gradiente (Comisión Nacional del Agua, 2010) (Tabla 2). Basados en la clasificación de Miranda y Hernández-X. (1963), a lo largo del gradiente se encuentran seis diferentes tipos de vegetación natural: (i) bosque de Abies (3,600-3,500 m), (ii) bosque de Pinus (3,500-2,800 m), (iii) bosque de Pinus-Quercus (2,800$2,400 \mathrm{~m}$ ), (iv) bosque mesófilo de montaña $(2,400-1,300$ $\mathrm{m})$, (v) encinares tropicales $(1,300-500 \mathrm{~m})$ y (vi) selva mediana subcaducifolia (500 $\mathrm{m}$ hasta el nivel del mar). Estos tipos de vegetación forman parte de un mosaico paisajístico que comprende fragmentos aislados de vegetación natural o bosque maduro (BM), zonas con vegetación azonal (VA) en cañadas y al lado de ríos (vegetación ribereña), así como sitios con diferentes grados de perturbación antrópica, como son el bosque perturbado (BP), el cual es un ambiente sometido a tala parcial, impacto por animales (cabras, borregos, vacas), eliminación del sotobosque, etc., y la vegetación secundaria (VS), que comprende bosques secundarios en etapas intermedias de sucesión (15-25 años).

Trabajo de campo. El muestreo de los helechos y licófitos se realizó entre los meses de febrero del 2012 y enero del
2014. A lo largo del transecto se establecieron de 15 a 25 parcelas no permanentes de $20 \times 20 \mathrm{~m}$ (Kessler y Bach, 1999) en cada uno de los ocho sitios de estudio ubicados en diferentes pisos altitudinales, separados por $500 \mathrm{~m}$ de altitud (20-500 m, 500-1,000 m, 1,000-1,500 m, 1,500-2,000 m, 2,000-2,500 m, 2,500-3,000 m, 3,000-3,500 m, 3,500-3,600 $\mathrm{m}$; Figura 1). Para simplificar, en el texto, se menciona solo el límite altitudinal inferior de cada piso. En cada una de las elevaciones y en los diferentes tipos de vegetación presentes en los pisos altitudinales, las parcelas fueron distribuidas por igual en diferentes ambientes naturales y derivados de la acción humana, es decir, se establecieron cinco en bosque maduro, cinco en bosque perturbado y cinco en zonas con vegetación secundaria. Además en los pisos de 500 m, 1,500 m y 2,000 m existen cañadas y vegetación ribereña donde también se estableció la misma cantidad de parcelas por ambiente, en el resto de los pisos altitudinales estudiados no se encuentra esta vegetación azonal.

En cada una de las parcelas se registró la presencia/ausencia de las especies terrestres y además las epífitas del sotobosque, es decir las plantas que crecen sobre los troncos de los árboles grandes y en árboles jóvenes o arbustos hasta una altura aproximada de $8 \mathrm{~m}$ (Krömer et al., 2007), las cuales fueron colectadas mediante una garrocha. La colecta de muestras botánicas se realizó en cada piso altitudinal y de acuerdo a Lot y Chiang (1986). Los ejemplares fueron identificados con base en Mickel y Smith (2004), comparaciones con ejemplares de herbario, y la consulta a especialistas (ver agradecimientos). La clasificación taxonómica 
Tabla 1. Familias y géneros de helechos y licófitos con mayor representatividad a lo largo del gradiente altitudinal del Cofre de Perote, centro de Veracruz, México.

\begin{tabular}{|c|c|c|c|c|c|}
\hline Familia & No. de especies & $\%$ & Género & No de especies & $\%$ \\
\hline Polypodiaceae & 33 & 21.3 & Asplenium & 12 & 7.7 \\
\hline Dryopteridaceae & 24 & 15.5 & Elaphoglossum & 11 & 7.1 \\
\hline Pteridaceae & 20 & 12.9 & Pleopeltis & 9 & 5.8 \\
\hline Aspleniaceae & 13 & 8.4 & Blechnum & 8 & 5.2 \\
\hline Hymenophyllaceae & 11 & 7.1 & Polypodium & 8 & 5.2 \\
\hline Blechnaceae & 8 & 5.2 & Hymenophyllum & 6 & 3.9 \\
\hline Dennstaedtiaceae & 7 & 4.5 & Selaginella & 6 & 3.9 \\
\hline Thelypteridaceae & 6 & 3.9 & Thelypteris & 5 & 3.2 \\
\hline Woodsiaceae & 6 & 3.9 & Adiantum & 5 & 3.2 \\
\hline Lycopodiaceae & 5 & 3.2 & Diplazium & 4 & 2.6 \\
\hline
\end{tabular}

se realizó de acuerdo a Mickel y Smith (2004) y Smith et al. (2006), las actualizaciones nomenclaturales se uniformizaron según los siguientes autores: Ranker et al. (2004), Ebihara et al. (2006), Kessler et al. (2011b), Li et al. (2012), Øllgaard (2012), Grusz y Windham (2013) y Smith y Tejero-Díez (2014). Los duplicados fueron depositados en el Herbario Nacional (MEXU) del Instituto de Biología de la UNAM, así como parcialmente en los herbarios de la Universidad Veracruzana (CIB), de la Universidad de California (UC), Berkeley, E.E. UU y/o del Instituto de Ecología, A.C. (XAL).

Análisis de datos. Se presenta un listado de especies que incluye datos referentes al rango altitudinal, hábito (terrestre, epífito, rupícola y escandente) y tipo de vegetación donde fueron registradas (Apéndice 1). Se señalan también las especies que se encuentran protegidas por la NOM-059-SEMARNAT-2010, los nuevos registros para el estado y las especies endémicas de Veracruz y/o México, así como el número de parcelas en las cuales fue encontrado cada taxón. Se utilizó el estimador Chao 2 obtenido en el programa Es-
timateS 9.1.0 (Colwell, 2013) para conocer el número total de especies estimadas en cada piso altitudinal.

\section{Resultados}

En total, para las 135 parcelas muestreadas a lo largo del gradiente altitudinal, se registraron 140 especies y cuatro variedades de helechos agrupadas en 59 géneros y 22 familias, así como 11 especies de licófitos distribuidas en tres géneros y dos familias (Apéndice 1). Los géneros con un mayor número de especies fueron: Asplenium L. (12 spp.), Elaphoglossum Schott ex J.Sm. (11 spp.) y Pleopeltis Humb. \& Bonp. ex. Willd (9 spp.). La familia Polypodiaceae es la más rica con 33 especies, seguida de Dryopteridaceae (24) y Pteridaceae (20) (Tabla 1). Se registraron 79 especies terrestres y 65 epífitas, siete rupícolas, dos terrestres o rupícolas, una terrestre o epífita y una escandente. Dos especies del género Elaphoglossum -Elaphoglossum pallidum C. Chr. y Elaphoglossum squarrosum (K1.) T. Moore- y Polystichum muricatum L. Fée son nuevos registros para el

Tabla 2. Riqueza de especies de helechos y licófitos por piso altitudinal (Alt) y tipo de vegetación (TV): selva mediana subcaducifolia (SMS), encinar tropical (ET), bosque mesófilo de montaña (BMM), bosque de Pinus-Quercus (BPQ), bosque de Pinus (BP) y bosque de Abies (BA), en diferentes ambientes naturales y derivados de la acción antrópica: bosque maduro (BM), bosque perturbado (BP), vegetación secundaria (VS) y vegetación azonal (VA; ríparia y cañadas). Se indica el número de especies totales, de epífitas (Epi) y terrestres (Ter), así como la riqueza estimada (Est) por Chao2 y el porcentaje que representan las especies observadas con respecto a las estimadas (\%). Se presentan datos de temperatura (T) y precipitación anual (Prec).

\begin{tabular}{|c|c|c|c|c|c|c|c|c|c|c|c|}
\hline \multirow[b]{2}{*}{$\begin{array}{l}\text { Sitio (No. } \\
\text { de parcelas) }\end{array}$} & \multirow[b]{2}{*}{ Alt (m) } & \multirow[b]{2}{*}{ TV } & \multirow[b]{2}{*}{$\mathrm{T}\left({ }^{\circ} \mathrm{C}\right)$} & \multirow[b]{2}{*}{$\begin{array}{l}\text { Prec } \\
(\mathbf{m m})\end{array}$} & \multirow[b]{2}{*}{ BM } & \multirow[b]{2}{*}{ BP } & \multirow[b]{2}{*}{ VS } & \multicolumn{2}{|c|}{ No de especies } & \multirow[b]{2}{*}{$\begin{array}{l}\text { Est } \\
\text { (Chao2) }\end{array}$} & \multirow[b]{2}{*}{$\%$} \\
\hline & & & & & & & & VA & $\begin{array}{l}\text { Total } \\
\text { (Epi/Ter) }\end{array}$ & & \\
\hline La Mancha (15) & 20 & SMS & 25 & 800 & 3 & 3 & 3 & - & $4(2 / 2)$ & 4 & 100 \\
\hline Palmarejo (15) & 500 & SMS/ET & 21 & 900 & 11 & 7 & 7 & 26 & $28(5 / 20)$ & 35.5 & 78 \\
\hline Chavarrillo (15) & 1,000 & ET & 21 & 900 & 7 & 7 & 8 & - & $14(8 / 6)$ & 21.3 & 65 \\
\hline Los Capulines (25) & 1,500 & BMM & 19 & 1,800 & 44 & 23 & 22 & 44 & $66(34 / 32)$ & 67.6 & 97 \\
\hline El Zapotal (15) & 2,000 & BMM & 18 & 1,800 & 46 & 35 & 29 & - & $62(31 / 30)$ & 73.5 & 84 \\
\hline El Encinal (20) & 2,500 & BPQ & 12 & 1,900 & 39 & 35 & 32 & 31 & $46(24 / 21)$ & 46.7 & 98 \\
\hline Los Pescados (15) & 3,000 & $\mathrm{BP}$ & 10 & 800 & 7 & 6 & 6 & - & $9(3 / 6)$ & 9 & 100 \\
\hline El Conejo (15) & 3,500 & $\mathrm{BA}$ & 9 & 800 & 4 & 3 & 7 & - & $7(1 / 6)$ & 7 & 100 \\
\hline
\end{tabular}


estado de Veracruz, las cuales se registraron en el bosque de Pinus-Quercus a una elevación de 2,500 m. De la lista total de especies, nueve están protegidas por la NOM-059SEMARNAT-2010, éstas en su mayoría (7 spp.) se encontraban en el bosque mesófilo de montaña y la vegetación de Pinus-Quercus, de las cuales cinco corresponden a helechos arborescentes. Los valores del estimador Chao 2 muestran que se tiene entre el 77 y $100 \%$ de las especies estimadas en cada uno de los pisos altitudinales, sin embargo en el piso de 1,000 m se obtuvo apenas el $62 \%$ (Tabla 2).

La riqueza de especies fue contrastante en diferentes pisos altitudinales del gradiente, ya que en elevaciones medias $(1,500$ a 2,500 m) se presentaron los valores máximos, mientras que los extremos superior e inferior del gradiente contienen muy pocas especies. Los tipos de vegetación con mayor registro de helechos y licófitos fueron el bosque mesófilo de montaña (con 96 especies), seguido del bosque de Pinus-Quercus (46 spp.) y la selva mediana subcaducifolia presente a $500 \mathrm{~m}$ (28 spp.). En la zona que se encuentra cercana al nivel del mar, así como en las partes altas del Cofre de Perote, la riqueza disminuyó hasta un 98 y $96 \%$ respectivamente (Tabla 2 ).

Con respecto a la relación de especies epífitas y terrestres a lo largo del gradiente altitudinal, se observó que en las elevaciones medias de 1,000 a 2,500 m, la proporción de estas se mantiene entre el 47 y $52 \%$ en relación a la riqueza de cada piso altitudinal, mientras que hacia los extremos del gradiente, los helechos y licófitos epífitos disminuyeron fuertemente; sin embargo, en La Mancha (20 m) esta proporción se encontró en un $50 \%$, aunque en base de un número de especies muy reducido (Tabla 2). Los bosques maduros en las elevaciones medias fueron los ambientes con la mayor cantidad de especies en comparación con la vegetación derivada de la acción antrópica, mientras que en los extremos del gradiente los valores de riqueza fueron similares en bosques naturales y perturbados. Se destaca el hecho de que en los pisos donde existe vegetación azonal, la cantidad de especies registradas fue mayor si se compara con el resto de los ambientes, incluso con el bosque maduro (Tabla 2).

\section{Discusión}

Riqueza de especies. La riqueza de 155 taxa registrados en este trabajo, representa el 27,5\% de los helechos y licófitos de Veracruz (Tejero-Díez et al., 2011; Krömer et al., 2015) y el $15 \%$ de las especies de México (Mickel y Smith, 2004, Tejero-Díez et al., 2014). Considerando que esta riqueza se registró en una superficie de muestra pequeña, el gradiente altitudinal estudiado resguarda una importante proporción de la pteridoflora del país. Los valores de las especies observadas son cercanos a las estimadas por Chao2 (en general más del $80 \%$ ), lo cual indica un muestreo satisfactorio (Tabla 2), únicamente en el encinar tropical (500 y 1,000 m) se
Tabla 3. Riqueza de especies de helechos y licófitos registrada en gradientes altitudinales ubicados en diferentes regiones del Neotrópico, indicando su rango altitudinal y latitud.

\begin{tabular}{|c|c|c|c|c|}
\hline Región, País & $\begin{array}{l}\text { Elevación } \\
\text { (m) }\end{array}$ & Latitud & $\begin{array}{l}\text { No de } \\
\text { especies }\end{array}$ & Autores \\
\hline $\begin{array}{l}\text { Parque Nacional } \\
\text { Carrasco, Bolivia }\end{array}$ & $300-3,950$ & $17^{\circ} 26^{\prime} \mathrm{S}$ & 473 & $\begin{array}{l}\text { Kessler et } \\
\text { al.,1999 }\end{array}$ \\
\hline $\begin{array}{l}\text { Yungas de La Paz, } \\
\text { Bolivia }\end{array}$ & $350-4,000$ & $16^{\circ} 13^{\prime} \mathrm{S}$ & $\begin{array}{l}264 \text { (solo } \\
\text { epífitas) }\end{array}$ & $\begin{array}{l}\text { Krömer et } \\
\text { al., } 2005\end{array}$ \\
\hline Napo, Ecuador & $400-4,000$ & $0^{\circ} 20^{\prime} \mathrm{S}$ & 350 & $\begin{array}{l}\text { Salazar et } \\
\text { al., } 2015\end{array}$ \\
\hline Pichincha, Ecuador & $450-3,600$ & $0^{\circ} 09^{\prime} \mathrm{N}$ & 319 & $\begin{array}{l}\text { Salazar et } \\
\text { al., } 2015\end{array}$ \\
\hline $\begin{array}{l}\text { La Selva-Volcán } \\
\text { Barva, Costa Rica }\end{array}$ & $30-2,300$ & $10^{\circ} 08^{\prime} \mathrm{N}$ & 246 & $\begin{array}{l}\text { Watkins et } \\
\text { al., 2006a }\end{array}$ \\
\hline $\begin{array}{l}\text { Braulio Carrillo-La } \\
\text { Selva y Cerro de la } \\
\text { Muerte, Costa Rica }\end{array}$ & $70-3,400$ & $10^{\circ} 24^{\prime} \mathrm{N}$ & 484 & $\begin{array}{l}\text { Kluge y } \\
\text { Kessler, } \\
2005\end{array}$ \\
\hline $\begin{array}{l}\text { Centro de } \\
\text { Veracruz, México }\end{array}$ & $20-3,500$ & $19^{\circ} 31^{\prime} \mathrm{N}$ & 155 & $\begin{array}{l}\text { Este } \\
\text { estudio }\end{array}$ \\
\hline
\end{tabular}

obtuvieron valores por debajo del $80 \%$ de las especies estimadas, debido al alto número de taxa únicos y duplicados. Estos bajos valores pueden explicarse por la homogeneidad de microambientes y la poca disponibilidad de nichos en el encinar tropical, lo que limita la presencia de especies (Kluge y Kessler, 2011), caso contrario a lo que sucede en el bosque mesófilo de montaña, en donde el estimador indica que las especies observadas representan más del $80 \%$ de las estimadas.

Campos-Salas (2011) registra para un gradiente altitudinal de 1,500-3,700 m ubicado en el estado de México una riqueza de 125 helechos y licófitos, cifra que coincide con el número de especies registradas en el presente estudio en esa franja altitudinal (126 especies entre 1,500 y 3,500 m). Krömer et al. (2013b), en un gradiente altitudinal de 140-1,650 $\mathrm{m}$ en la región de Los Tuxtlas, Veracruz, registraron 108 especies y no incluyeron vegetación perturbada; esta riqueza es mayor si se compara con la registrada en el presente estudio (99 especies entre $20 \mathrm{~m}$ y 1,500 m). Esto debido a que Los Tuxtlas es un área con una elevada precipitación (> $4,000 \mathrm{~mm}$ ) y humedad ambiental, factores que favorecen una alta riqueza de helechos (Krömer et al., 2013b; Acebey et al., 2015). Por otro lado, Hietz y Hietz-Seifert (1995) registraron 33 especies de helechos epífitos en árboles mayores a $10 \mathrm{~cm}$ de diámetro que fueron muestreados en fragmentos de bosque natural, ubicados entre los 720-2,370 m en el mismo gradiente altitudinal del presente estudio; sin embargo, dicha riqueza epifítica es menor a la registrada en este trabajo (61 spp.) entre 500-2,500 m, lo que confirma la importancia del sotobosque como hábitat para los helechos epífitos (Krömer et al., 2007).

Los datos de la alta riqueza de especies presentados, muestran la importancia de este gradiente por su gran aporte 
a la pteridoflora a nivel regional. Sin embargo, si contrastamos estos datos con otras zonas del Neotrópico cercanas al Ecuador resultan notablemente menores (Tabla 3). Esto coincide con el gradiente de diversidad latitudinal, en el cual el número de especies de helechos y licófitos por unidad de área aumenta conforme se acerca al Ecuador y se aleja de los polos (Moran, 2008; Salazar et al., 2015), aunque los estudios realizados en Bolivia entre $14-16^{\circ}$ de latitud Sur demuestran la extraordinaria riqueza de los bosques húmedos de los Andes (Krömer et al., 2005; Kessler et al., 2011a).

En el presente estudio, se observó que las elevaciones medias (1,500-2,500 m) son las zonas con mayor riqueza de especies, situación que coincide con lo reportado en otros países Neotropicales (Salazar et al., 2015). En esta franja altitudinal, se encuentra el bosque mesófilo de montaña el cual es el tipo de vegetación más diverso por unidad de superficie en México y en el cual los helechos y licófitos son un componente conspicuo (Rzedowski, 1996, Tejero-Díez et al., 2014). En este tipo de vegetación predominan las condiciones climáticas de temperaturas templadas y alta humedad ambiental que favorecen el desarrollo de una gran riqueza de helechos (Kluge et al., 2006; Watkins et al., 2006a; Salazar et al., 2015). De acuerdo a Tejero-Díez et al. (2011) el bosque de Pinus-Quercus es otro tipo de vegetación con alta riqueza de helechos en Veracruz, y en concordancia en este estudio fue el segundo más diverso. Por otro lado, los bosques de coníferas y los encinares tropicales fueron los tipos de vegetación con menor número de especies, esto se debe a que la sequía estacional y los climas cálido-secos en la parte baja y fríos en la parte alta del gradiente limitan la presencia de helechos (Tejero-Díez et al., 2011), ya que en estos extremos del gradiente sobreviven únicamente las especies que desarrollan estrategias fisiológicas como la tolerancia a la desecación, poiquilohidría o fotoinhibición a través del desarrollo de escamas y tricomas (Watkins et al., 2006b; Hietz, 2010).

El componente epifítico representa casi el $50 \%$ de la riqueza total registrada, sin embargo su proporción cambia de acuerdo a la altitud. En la franja media del gradiente $(1,000-$ 2,500 m), las epífitas están representadas con cerca del $50 \%$ del total de especies registradas en cada piso altitudinal, mientras que los valores decrecen en los extremos del gradiente. Esto coincide con los resultados obtenidos en la región de Los Tuxtlas por Acebey et al. (2015), en donde la riqueza de especies terrestres es mayor en las selvas tropicales de las zonas bajas y las epífitas son más numerosas en el bosque mesófilo de montaña de elevación media. Lo anterior indica que las condiciones de sequía y temperaturas extremas presentes en los límites superior e inferior del gradiente, limitan a los helechos y licófitos en general y específicamente a los epífitos (Kessler, 2001b; Krömer et al., 2005).

Riqueza por tipo de hábitat. Los ambientes con bosque maduro entre los 1,500-2,500 m, contienen la mayor cantidad de especies, mientras que el bosque perturbado y las zonas con vegetación secundaria de esta franja muestran valores más bajos. Lo anterior indica que las modificaciones en la estructura de los bosques húmedos causados por la acción humana afectan de manera considerable a las especies de helechos y licófitos, ya que al existir cambios en el microclima y fisonomía del bosque como producto de la perturbación (Barthlott et al., 2001; Krömer y Gradstein, 2003; Köster et al., 2009), la riqueza de éstas se pierde desde el 40 hasta el $70 \%$. Esta pérdida pudiera deberse a la vulnerabilidad de las especies higrófilas y umbrófilas que habitan principalmente en el sotobosque de los bosques maduros en relación al microclima más seco y caliente causado por la mayor entrada de luz en los ambientes antropizados (Acebey et al., 2003; Paciencia y Prado, 2005; Krömer et al., 2007; Larrea y Werner, 2010; Carvajal-Hernández et al., 2014). Sin embargo, en los pisos altitudinales de 1,000 y $3,500 \mathrm{~m}$, la vegetación secundaria contiene una riqueza similar a la del bosque maduro, debido a la presencia de especies adaptadas a condiciones extremas de sequía y temperatura, las cuales no se ven afectadas por la perturbación (Hietz, 2010). Como ejemplos de lo anterior se destacan las especies de Adiantum L., Anemia Sw. y Myriopteris Fée en 1,000 m, los cuales son comunes en bosques secos y presentan rizomas subterráneos capaces de sobrevivir al fuego; por otro lado, en 3,500 $\mathrm{m}$ se encuentran especies de Pleopeltis y Polystichum Roth, que como estrategia adaptativa crecen sobre o entre rocas y presentan escamas y abundantes tricomas que les permiten tolerar altos niveles de radiación (Hietz, 2010).

En la vegetación azonal (cañadas y al lado de ríos), la riqueza de helechos y licófitos encontrada es similar a la de los bosques maduros, incluso en ocasiones es mayor. Para el caso del piso de $500 \mathrm{~m}$, la riqueza registrada en vegetación ribereña fue el doble de la que se observó en selva mediana subcaducifolia y encinar tropical maduro, destacándose que la mayoría de las especies adicionales son terrestres o rupícolas. Lo anterior concuerda con lo encontrado por Kessler (2001a) en Bolivia y por Kluge y Kessler (2011) en Costa Rica, donde registran una mayor riqueza de especies en las orillas de ríos y confirman la preferencia de los helechos por habitar en zonas con influencia de agua y humedad ambiental alta.

Nuevos registros y conservación. A pesar de que el estado de Veracruz en el pasado y recientemente ha sido motivo de diferentes estudios sobre la riqueza y distribución de helechos y licófitos (p. ej., Lira y Riba, 1984; Palacios-Ríos, 1992; Tejero-Díez et al., 2011; Carvajal-Hernández et al., 2014; Acebey et al., 2015), aún es posible aportar nuevas contribuciones a la pteridoflora del estado. Los tres nuevos registros del presente estudio pertenecen a los géneros Elaphoglossum y Polystichum, los cuales fueron encontrados en el bosque de Pinus-Quercus en la franja altitudinal de 2,500 m. El primero de ellos, E. pallidum se distribuye en 
Helechos y Licófitos del Cofre de Perote, Veracruz

bosque mesófilo y bosque de Pinus-Quercus en cinco estados (Chiapas, Oaxaca, Guerrero, Michoacán y Morelos); el segundo, E. squarrosum estaba registrado en los estados de Chiapas, Oaxaca, Guerrero y México, en bosques húmedos montañosos, mientras que Polystichum muricatum se tenía reportado en los estados de Oaxaca y Chiapas en altitudes menores (1,350-1,850 m). El hallazgo de estas especies en Veracruz, aumenta el número de taxa de helechos y licófitos registrados y confirmados a 564 (Krömer et al., 2015) y reafirma la necesidad de continuar con la elaboración de inventarios florísticos en general y estudios de la pteridoflora en particular, sobre todo en zonas poco exploradas como la Sierra de Huayacocotla y la Sierra de Otontepec al norte del estado, así como en zonas con cañadas que por su difícil acceso han sido poco estudiadas.

El gran número de especies de helechos y licófitos registrado, confirma que la región montañosa del centro de Veracruz es una de las de mayor riqueza de estos grupos en el estado (Tejero-Díez et al., 2011). Además se documenta la disminución de especies en ambientes sometidos a perturbación antrópica y la alta riqueza de helechos en vegetación azonal. Debido a que todos los tipos de vegetación natural presentes a lo largo del gradiente altitudinal se encuentran fuertemente alterados (Ellis et al., 2011), la situación de muchos helechos y licófitos en la zona es vulnerable, sobre todo de las especies protegidas por la NOM-059-SEMARNAT-2010 y endémicas de Veracruz (Asplenium venturae A.R.Sm. y Cibotium schiedei Schltdl. \& Cham.), las cuales son de distribución restringida en el centro del estado. Sumado a estas, se encuentran las especies de Hymenophyllaceae, Phlegmariurus (Herter) Holub y helechos gramitidoides que se encuentran principalmente en el bosque mesófilo y se ha demostrado su vulnerabilidad cuando existen cambios en el ambiente provocados por las actividades antrópicas (Tejero-Díez et al., 2011; Gehrig-Downie et al., 2012; Krömer et al., 2013a, Armenta-Montero et al., 2015). Sin embargo, la vegetación azonal representa un reservorio importante de la pteridoflora, por lo tanto la conservación de fragmentos de estos ambientes en Veracruz y México, puede y debería considerarse en futuros planes de conservación.

\section{Agradecimientos}

Agradecemos a Jorge Gómez-Díaz, Valeria Guzmán-Jacob y Samaria Armenta-Montero por su apoyo en campo, a esta última se agradece además la realización del mapa. A Amparo R. Acebey, Juan C. López-Acosta y Michael Kessler por sus comentarios útiles para el enriquecimiento del documento. Al Instituto de Ecología, A.C. por su autorización para realizar el muestreo en "La Mancha". A Alan R. Smith (UC-Berkeley) y Robin C. Moran (NY Botanical Garden) por la corroboración de las identificaciones del material de herbario. A los dos revisores anónimos por las sugerencias que permitieron mejoras sustanciales del manuscrito. Al CONACyT por la beca (No. 224291) otorgada al primer autor.

\section{Literatura citada}

Acebey A., Gradstein S.R. y Krömer T. 2003. Species richness and habitat diversification of bryophytes in submontane rain forest and fallows of Bolivia. Journal of Tropical Ecology 19:9-18.

Acebey A.R., Krömer T., Vázquez-Torres M. y Tejero-Díez J.D. 2015. Helechos y licófitos de la Reserva de la Biosfera Los Tuxtlas, Veracruz, México. Botanical Sciences 93:313-344.

Armenta-Montero S., Carvajal-Hernández C., Ellis E.A. y Krömer T. 2015. Distribution and conservation status of Phlegmariurus (Lycopodiaceae) in the state of Veracruz, Mexico. Tropical Conservation Science 8:114-137

Arriaga L., Espinoza J.M., Aguilar C., Martínez E., Gómez L. y Loa E. Coord. 2000. Regiones Terrestres Prioritarias de México. Comisión Nacional para el Conocimiento y uso de la Biodiversidad. México, D.F. <http://www.conabio.gob.mx/conocimiento/regionalizacion/doctos/Tsureste.html> (consultado el 25 febrero 2014).

Barthlott W., Schmit-Neuerburg V., Nieder J. y Engwald S. 2001. Diversity and abundance of vascular epiphytes: a comparison of secondary vegetation and primary montane rain forest in the Venezuelan Andes. Plant Ecology 152:145-156.

Campos-Salas N. 2011. Diversidad y distribución de helechos y licopodios en la cuenca alta del río Chiquihuitero, Coatepec Harinas, México, México. Tesis de Licenciatura, Facultad de Estudios Superiores Iztacala, Universidad Nacional Autonóma de México. Tlalnepantla, México. 75 pp.

Carvajal-Hernández C., Krömer T. y Vázquez-Torres M. 2014. Riqueza y composición florística de pteridobiontes en bosque mesófilo de montaña y ambientes asociados, en el centro de Veracruz, México. Revista Mexicana de Biodiversidad 85:491-501.

Castillo-Campos G., Avendaño-Reyes S. y Medina-Abreo M.E. 2011. Flora y vegetación. En: Comisión Nacional para el Conocimiento y Uso de la Biodiversidad (CONABIO). La Biodiversidad en Veracruz: Estudio de Estado. Volumen I. pp. 163-179, Comisión Nacional para el Conocimiento y Uso de la Biodiversidad, Gobierno del Estado de Veracruz, Universidad Veracruzana, Instituto de Ecología, A. C. México.

Colwell, R. K. 2013. EstimateS: Statistical estimation of species richness and shared species from samples. Version 9. <http:// purl.oclc.org/estimates.> (consultado el 22 de enero de 2014).

Comisión Nacional del Agua. 2010. Normales climatológicas por estación. <http://smn.cna.gob.mx./index.php?option=com_con tent\&view=article\&id=42\&Itemid=75>. $($ consultado el 17 de octubre de 2012).

CONABIO. 2010. El Bosque Mesófilo de Montaña en México: Amenazas y Oportunidades para su Conservación y Manejo Sostenible. Comisión Nacional para el Conocimiento y Uso de la Biodiversidad. México, D.F.

Ebihara A., Dubuisson J-Y., Iwatsuki K., Hennequin S. e Ito M. 2006. A taxonomic revision of Hymenophyllaceae. Blumea 51:221-280.

Ellis E.A., Martínez-Bello M. y Monroy-Ibarra R. 2011. Focos rojos para la conservación de la biodiversidad. En: Comisión 
Nacional para el Conocimiento y Uso de la Biodiversidad (CONABIO). La Biodiversidad en Veracruz: Estudio de Estado. Volumen I, pp. 351-367, Comisión Nacional para el Conocimiento y Uso de la Biodiversidad, Gobierno del Estado de Veracruz, Universidad Veracruzana, Instituto de Ecología, A. C. México.

Gehrig-Downie C., Marquardt J., Obregón A., Bendix J. y Gradstein S.R. 2012. Diversity and vertical distribution of filmy ferns as a tool for identifying the novel forest type "tropical lowland cloud forest". Ecotropica 18:35-44.

Geissert-Kientz D. y Enríquez-Fernández E. 2011.Geomorfología. En: Comisión Nacional para el Conocimiento y Uso de la Biodiversidad (CONABIO). La Biodiversidad en Veracruz: Estudio de Estado. Volumen I, pp. 53-68, Comisión Nacional para el Conocimiento y Uso de la Biodiversidad, Gobierno del Estado de Veracruz, Universidad Veracruzana, Instituto de Ecología, A. C. México.

Gómez-Díaz J.A. 2010. Comparación florística de epífitas vasculares entre un bosque mesófilo de montaña y un acahual en el municipio de Tlalnelhuayocan, Veracruz. Tesis de Licenciatura, Facultad de Biología, Universidad Veracruzana. Xalapa, Veracruz. 117 pp.

Gómez-Pompa A. 2010. Programa Flora de Veracruz. En: GómezPompa A., Krömer T. y Castro-Cortés R. Coord. Atlas de la Flora de Veracruz: Un Patrimonio Natural en Peligro, pp. 4356, Comisión del Estado de Veracruz para la Conmemoración de la Independencia Nacional y la Revolución Mexicana, Xalapa, México.

Grusz A.L. y Windham M.D. 2013. Toward a monophyletic Cheilanthes: The resurrection and recircumscription of Myriopteris (Pteridaceae). Phytokeys 32:49-64.

Hernández-Rojas A.C. 2010. Diversidad de helechos en bosques mesófilos con diferente dominancia arbórea en el centro de Veracruz, México. Tesis de Maestría, Instituto de Ecología A. C. Xalapa, Veracruz. 84 pp.

Hietz P. y Hietz-Seifert U.1995. Composition and ecology of vascular epiphyte communities along an altitudinal gradient in central Veracruz, Mexico. Journal of Vegetation Science 6:487498.

Hietz P. 2010. Fern adaptations to xeric environments. En: Mehltreter K., Walker L.R. y Sharpe J.M. Eds. Fern Ecology, pp.140176, Cambrige University Press, Cambridge, UK.

Kessler M. y Bach K. 1999. Using indicator groups for vegetation classification in species-rich Neotropical forests. Phytocoenologia 29:485-502.

Kessler M., Smith A.R. y González J. 1999. Inventario de pteridofítos en un transecto altitudinal en el Parque Nacional Carrasco, dpto, Cochabamba, Bolivia. Revista de la Sociedad Boliviana de Botánica 2:227-250.

Kessler M. 2001a. Patterns of diversity and range size of selected plant groups along an elevational transect in the Bolivian Andes. Biodiversity and Conservation 10:1897-1920.

Kessler M. 2001b. Pteridophyte species richness in Andean forests in Bolivia. Biodiversity and Conservation 10:1473-1495.

Kessler M., Kluge J., Hemp A. y Ohlemüller R. 2011a. A global comparative analysis of elevational species richness patterns of ferns. Global Ecology and Biogeography 20:868-880.

Kessler M., Moguel-Velázquez A.L., Sundue M. y Labiak P.H. 2011b. Alansmia, a new genus of grammitid ferns (Polypodiaceae) segregated from Terpsichore. Brittonia 63:233-244.

Kluge J. y Kessler M. 2005. Inventory of pteridophytes along an elevational transect in Braulio Carrillo National Park, La Selva Biological Station and Cerro de la Muerte, Costa Rica. Brenesia 63/64:11-34.

Kluge J., Kessler M. y Dunn R.R. 2006. What drives elevational patterns of diversity? A test of geometric constraints, climate and species pool effects for pteridophytes on an elevational gradient in Costa Rica. Global Ecology and Biogeography 15:358-371.

Kluge J. y Kessler M. 2011. Influence of niche characteristics and forest type on fern species richness, abundance and plant size along an elevational gradient in Costa Rica. Plant Ecology 212:1109-1121.

Köster N., Friedrich K., Nieder J. y Barthlott W. 2009. Conservation of epiphyte diversity in an Andean landscape transformed by human land use. Conservation Biology 23:911-919.

Krömer T. y Gradstein S.R. 2003. Species richness of vascular epiphytes in two primary forests and fallows in the Bolivian Andes. Selbyana 24:190-195.

Krömer T., Kessler M., Gradstein S.R. y Acebey A. 2005. Diversity patterns of vascular epiphytes along an elevational gradient in the Andes. Journal of Biogeography 32:1799-1809.

Krömer T., Kessler M. y Gradstein S.R. 2007. Vertical stratification of vascular epiphytes in submontane and montane forest of the Bolivian Andes: the importance of the understory. Plant Ecology 189:261-278.

Krömer T., Acebey A.R. y Smith A.R. 2013a. Taxonomic update, distribution and conservation status of grammitid ferns (Polypodiaceae, Polypodiopsida) in Veracruz State, Mexico. Phytotaxa 82:29-44.

Krömer T., Acebey A., Kluge J. y Kessler M. 2013b. Effects of altitude and climate in determining elevational plant species richness patterns: A case study from Los Tuxtlas, Mexico. Flora 208:197-210.

Krömer T., Carvajal-Hernández C., Acebey A.R. y Smith A.R. 2015. A decade of new pteridophyte records for the State of Veracruz, Mexico. American Fern Journal 105:162-175.

Kreft H., Jetz W., Mutke J. y Barthlott W. 2010. Contrasting environmental and regional effects on global pteridophyte and seed plant diversity. Ecography 33:408-419.

Larrea M.L. y Werner F.A. 2010. Response of vascular epiphyte diversity to different land-use intensities in a neotropical montane wet forest. Forest Ecology and Management 260:1950-1955.

Li F-W. Pryer K.M y Windham M.D. 2012. Gaga, a new fern genus degregated from Cheilanthes (Pteridaceae). Systematic Botany 37:845-860.

Lira R. y Riba R. 1984. Aspectos fitogeográficos y ecológicos de la flora pteridofítica de la sierra de Santa Marta, Veracruz, México. Biótica 9:451-467.

Lot A. y Chiang F. 1986. Manual de Herbario. Administración y Manejo de Colecciones, Técnicas de Recolección y Preparación de Ejemplares Botánicos. Consejo Nacional de la Flora de México, A.C., México, D.F.

Magaña P. y Villaseñor J.L. 2002. La flora de México ¿Se podrá conocer completamente? Ciencias 66:24-26.

Mickel J. y Smith A.R. 2004. The pteridophytes of Mexico. Memoirs of the New York Botanical Garden 88: 1-1054.

Miranda F. y Hernández-X. E. 1963. Los tipos de vegetación de México y su clasificación. Boletín de la Sociedad Botánica de México 28:29-178.

Moran R.C. 1995. The importance of mountains to pteridophytes, with emphasis on Neotropical montane forests. En Churchill 
S.P., Balslev H., Forero E. y Luteyn J.L. Eds. Biodiversity and Conservation of Neotropical Montane Forest. Proceedings of a Symposium, The New York Botanical Garden, 21-26 June 1993. pp. 359-363, The New York Botanical Garden, Nueva York.

Moran R.C. 2008. Diversity, biogeography, and floristics. En. Ranker T.A. y Haufler C.H. Eds. Biology and Evolution of Ferns and Lycophytes, pp 367-394, Cambridge University Press, Nueva York.

Øllgaard B. 2012. New combinations in Neotropical Lycopodiaceae. Phytotaxa 57:10-22.

Paciencia M.L.B. y Prado J. 2005. Effects of forest fragmentation on pteridophyte diversity in a tropical rain forest in Brazil. Plant Ecology 180:87-104.

Palacios-Ríos M. 1992. Las pteridofitas del Estado de Veracruz. Tesis de Maestría, Facultad de Ciencias, Universidad Nacional Autónoma de México. México, D.F. 364 pp.

Ranker T.A., Smith A.R., Parris B.S., Geiger J.M.O, Haufler C.H., Straub S.C.K. y Schneider H. 2004. Phylogeny and evolution of grammitid ferns (Grammitidaceae): A case of rampant morphological homoplasy. Taxon 53:415-428.

Rzedowski J. 1996. Análisis preliminar de la flora vascular de los bosques mesófilos de montaña de México. Acta Botanica Mexicana 35:25-44.

Salazar-Rodríguez J.L. 2010. Holoepífitas vasculares del bosque mesófilo de montaña del municipio de Acajete, Veracruz. Tesis de Licenciatura, Facultad de Biología, Universidad Veracruzana. Xalapa, Veracruz. 42 pp.

Salazar L., Homeier J., Kessler M., Abrahamczyk S., Lehnert M., Krömer T. y Kluge J. 2015. Diversity patterns of ferns along elevation in Andean tropical forests. Plant Ecology and Diversity 8:13-24.

Smith A.R., Pryer K.M., Schuettpelz E., Korall P., Schneider H. y Wolf P.G. 2006. A classification for extant ferns. Taxon 55:705731.

Smith A.R. y Tejero-Díez J.D. 2014. Pleopeltis (Polypodiaceae), a redefinition of the genus and nomenclatural novelties. Botanical Sciences 92:43-58.

Soto-Esparza M. y Geissert K.D. 2011. Geografía. En: Comisión Nacional para el Conocimiento y Uso de la Biodiversidad (CO-
NABIO). La Biodiversidad en Veracruz: Estudio de Estado. Volumen I, pp. 31-34, Comisión Nacional para el Conocimiento y Uso de la Biodiversidad, Gobierno del Estado de Veracruz, Universidad Veracruzana, Instituto de Ecología, A.C. México D.F.

Soto-Esparza M. y Giddings-Berger L.E. 2011. Clima. En: Comisión Nacional para el Conocimiento y Uso de la Biodiversidad (CONABIO). La Biodiversidad en Veracruz: Estudio de Estado. Volumen I, pp. 35-52, Comisión Nacional para el Conocimiento y Uso de la Biodiversidad, Gobierno del Estado de Veracruz, Universidad Veracruzana, Instituto de Ecología, A. C. México.

Tejero-Díez D., Torres-Díaz A., Mickel J.T., Mehltreter V.K. y Krömer T. 2011. Helechos y licopodios. En: Comisión Nacional para el Conocimiento y Uso de la Biodiversidad (CONABIO). La Biodiversidad en Veracruz: Estudio de Estado. Volumen II. pp. 97-115. Comisión Nacional para el Conocimiento y Uso de la Biodiversidad, Gobierno del Estado de Veracruz, Universidad Veracruzana, Instituto de Ecología, A.C. México D.F.

Tejero-Díez J.D., Torres-Díaz A.N. y Gual-Díaz M. 2014. Licopodios y helechos en el bosque mesófilo de montaña de México. En: Gual-Díaz M. y Rendón-Correa A. Comp. Bosques Mesófilos de Montaña de México. Diversidad, Ecología y Manejo. pp. 197-220. Comisión Nacional para el Conocimiento y Uso de la Biodiversidad, México D.F.

Vázquez G.J.A. y Givnish T.J. 1998. Altitudinal gradients in tropical forest composition, structure and diversity in the Sierra de Manantlán. Journal of Ecology 86:999-1020.

von Humboldt A. 1822 (2004). Ensayo político sobre el Reino de la Nueva España. Porrúa. México, D.F. <https://archive.org/details/ensayopoliticos00arnagoog $>$ (consultado el 20 de febrero de 2014)

Watkins Jr. J.E., Cardelús C., Colwell R.K. y Moran R.C. 2006a. Species richness and distribution of ferns along an elevational gradient in Costa Rica. American Journal of Botany 93:73-83.

Watkins Jr. J.E., Kawahara A.Y., Leicht, S.A., Auld J.R., Bicksler A.J. y Kaiser K. 2006b. Fern laminar scales protect against photoinhibition from excess light. American Fern Journal 96:83-92.

Zamudio G. y Butanda A. 1999. Humboldt y la botánica americana. Ciencias 62:55-56.

Recibido: 16 de junio de 2014

Aceptado: 17 de agosto de 2014 
Apéndice 1. Relación de especies de helechos y licófitos registradas a lo largo del gradiente altitudinal del Cofre de Perote, centro de Veracruz, México. Colector: César I. Carvajal-Hernández (CICH); Herbario: MEXU: Instituto de Biología, UNAM; UC: Universidad de California-Berkeley; CIB: Instituto de Investigaciones Biológicas, Universidad Veracruzana y XAL: Instituto de Ecología, A.C.; Hábito (T: terrestre; E: epífito; R: rupícola; ES: escandente); Vegetación: tipo de vegetación (BA: bosque de Abies; BP: bosque de Pinus; BPQ: bosque de Pinus-Quercus; BMM: bosque mesófilo de montaña; ET: bosque tropical de Quercus o encinar tropical; SMS: selva mediana subcaducifolia); No.: número de parcelas donde la especie fue registrada; Observaciones (EV: endémica a Veracruz; EM: endémica a México; NR: nuevo registro para Veracruz; Categorías de riesgo de acuerdo a la NOM-059-Semarnat-2010: P: en peligro; Pr: sujeta a protección especial; A: amenazada).

\begin{tabular}{|c|c|c|c|c|}
\hline Familia/Especie (ejemplar de referencia, herbario) & Hábito & $\begin{array}{l}\text { Elevación } \\
(\mathrm{m} \text { s.n.m. })\end{array}$ & Vegetación & \\
\hline \multicolumn{5}{|l|}{ EQUISETOPSIDAE } \\
\hline \multicolumn{5}{|l|}{ LYCOPODIIDAE (Licófitos) } \\
\hline \multicolumn{5}{|l|}{ LYCOPODIACEAE } \\
\hline Lycopodium clavatum L. (CICH 357 MEXU) & $\mathrm{T}$ & 2,500 & $\mathrm{BPQ}$ & 6 \\
\hline Lycopodium thyoides Humb. \& Bonpl. ex Willd. (CICH 401 MEXU, UC) & $\mathrm{T}$ & 2,500 & $\mathrm{BPQ}$ & 1 \\
\hline Phlegmariurus linifolius (L.) B.Øllg. (CICH 795 CIB) & $\mathrm{E}$ & 2,000 & $\mathrm{BMM}$ & 1 \\
\hline $\begin{array}{l}\text { Phlegmariurus pringlei (Underw. \& F.E.Lloyd) B.Øllg. (CICH } 355 \\
\text { MEXU; CICH } 434 \text { MEXU, UC) }\end{array}$ & $\mathrm{E}$ & $1,500,2,500$ & $\mathrm{BMM}, \mathrm{BPQ}$ & 1 \\
\hline $\begin{array}{l}\text { Phlegmariurus taxifolius (Sw.) Á.Löve \& D.Löve (CICH } 353 \text { MEXU, } \\
\text { UC; CICH } 433 \mathrm{MEXU,} \mathrm{UC)}\end{array}$ & $\mathrm{E}$ & $1,500,2,500$ & $\mathrm{BMM}, \mathrm{BPQ}$ & 8 \\
\hline \multicolumn{5}{|l|}{ SELAGINELLACEAE } \\
\hline Selaginella harrisii Underw. \& Hieron. (CICH 506 MEXU, UC) & $\mathrm{T}$ & 500 & SMS & 1 \\
\hline Selaginella hoffmannii Hieron (CICH 530 CIB) & $\mathrm{T}$ & 500 & SMS & 1 \\
\hline Selaginella stellata Spring (CICH 430 MEXU, UC; CICH 659 MEXU) & $\mathrm{T}$ & $1,500,2,000$ & BMM & \\
\hline Selaginella stenophylla A.Braun (CICH 501 MEXU, UC) & $\mathrm{T}$ & 500 & SMS & \\
\hline Selaginella silvestris Aspl. (CICH 351 MEXU, UC) & $\mathrm{T}$ & 2,500 & $\mathrm{BPQ}$ & \\
\hline Selaginella pallescens (C.Presl) Spring (CICH 538 CIB) & $\mathrm{T}$ & 500 & SMS & \\
\hline
\end{tabular}

\section{POLYPODIIDAE (Helechos)}

ANEMIACEAE

Anemia adiantifolia (L.) Sw. (CICH485 CIB, ClCH536 MEXU)

ASPLENIACEAE

Asplenium auriculatum Sw. (CICH 439 MEXU, UC, CICH 641 ClB)

Asplenium blepharophorum Bertol. (CICH366 MEXU)

Asplenium castaneum Schltdl. \& Cham. (CICH 509 MEXU, CIB )

Asplenium cuspidatum Lam. (CICH 443 MEXU,UC)

Asplenium hallbergii Mickel \& Beitel (CICH 511 MEXU)

Asplenium harpeodes Kunze (CICH 449 MEXU, UC, CICH670 MEXU)

Asplenium miradorense Liebm. (CICH $482 \mathrm{MEXU})$

Asplenium monanthes L. (CICH 336 MEXU, UC; CICH 438 MEXU, UC)

Asplenium riparium Liebm. (CICH 674 MEXU, UC)

Asplenium serra Langsd. \& Fisch. (CICH 460 MEXU, CICH 643 MEXU)

Asplenium sessilifolium Desv var. sessilifolium (CICH 335 MEXU, UC)

Asplenium venturae A.R.Sm. (CICH 645 MEXU)

Schaffneria nigripes Fée (CICH 555 MEXU, CIB)

BLECHNACEAE

Blechnum appendiculatum Willd. (CICH 444 MEXU; $\mathrm{ClCH} 638$ MEXU)

Blechnum falciforme (Liebm.) C.Chr. (CICH 362 MEXU, CIB;

$\mathrm{ClCH} 649 \mathrm{CIB})$

Blechnum fragile (Liebm.) C.V.Morton \& Lellinger (CICH 450 MEXU;

$\mathrm{ClCH} 672 \mathrm{CIB}$ )

Blechnum gracile Kaulf. (CICH 540 MEXU, CIB)

$\mathrm{T}$

$$
500,1,000
$$

SMS, ET

10

E $1,500,2,000$

T 2,500

T 3,500

E 1,500

$\mathrm{T}$

T

E

$\mathrm{T}$

$3000,3,500$

$1,500,2,000$

1,500

T 1,500, 2,000,

$2,500,3,500$

Ty R 2,000

Ty $E \quad 1,500,2,000$

Ty R 2,500

T 2,000

R 500

$\mathrm{T}$

$1,500,2,000$

$2,000,2,500$

BMM

BMM

BPQ

BA

BMM

BA

(

(1)

1

(

$$
8
$$

Observaciones 
Apéndice 1. Continuación

\begin{tabular}{|c|c|c|c|c|c|}
\hline Familia/Especie (ejemplar de referencia, herbario) & Hábito & $\begin{array}{l}\text { Elevación } \\
\text { (m s.n.m.) }\end{array}$ & Vegetación & No. & $\begin{array}{l}\text { Observa- } \\
\text { ciones }\end{array}$ \\
\hline Blechnum occidentale L.(CICH 818 CIB) & $\mathrm{T}$ & 500 & SMS & 3 & \\
\hline Blechnum schiedeanum Hieron. (CICH 414 MEXU) & $\mathrm{T}$ & 1,500 & BMM & 13 & \\
\hline $\begin{array}{l}\text { Blechnum stoloniferum Mett. (CICH } 354 \text { MEXU, UC; } \\
\text { CICH } 671 \text { MEXU, CIB) }\end{array}$ & $\mathrm{T}$ & $2,000,2,500$ & $\mathrm{BMM}, \mathrm{BPQ}$ & 20 & \\
\hline $\begin{array}{l}\text { Blechnum wardiae Mickel \& Beitel (CICH } 478 \text { MEXU, UC; } \\
\text { CICH } 656 \text { MEXU, CIB) }\end{array}$ & $\mathrm{T}$ & $1,500,2,000$ & BMM & 6 & \\
\hline \multicolumn{6}{|l|}{ CIBOTIACEAE } \\
\hline \multicolumn{5}{|l|}{ CYATHEACEAE } & $\mathrm{EV}, \mathrm{P}$ \\
\hline Alsophila firma (Baker) D.S.Conant (CICH 411 MEXU, UC) & $\mathrm{T}$ & $1,500,2,000$ & BMM & 20 & $\operatorname{Pr}$ \\
\hline Cyathea bicrenata Liebm. (CICH 481MEXU, UC) & $\mathrm{T}$ & 1,500 & BMM & 6 & $\operatorname{Pr}$ \\
\hline Cyathea tuerckheimii Maxon (CICH 410 MEXU, UC) & $\mathrm{T}$ & $1,500,2,000$ & BMM & 29 & $\operatorname{Pr}$ \\
\hline \multicolumn{5}{|l|}{ DENNSTAEDTIACEAE } & $\operatorname{Pr}$ \\
\hline Dennstaedtia cicutaria (Sw.) T.Moore (CICH 531 CIB) & $\mathrm{T}$ & 500 & SMS & 1 & \\
\hline Dennstaedtia cornuta (Kaulf.) Mett. (CICH 480 MEXU, UC; CICH 699 CIB) & $\mathrm{T}$ & $1,500,2,000$ & $\mathrm{BMM}$ & 5 & \\
\hline Hypolepis blepharochlaena Mickel \& Beitel (CICH 349 MEXU, CIB) & $\mathrm{T}$ & 2,500 & BPQ & 5 & \\
\hline Hypolepis repens (L.) C.PresI (CICH 465 CIB, CICH 751 CIB ) & $\mathrm{T}$ & $1,500,2,000$ & BMM & 10 & \\
\hline Pteridium aquilinum var. feei (W.Schaffn. ex Fée) (CICH 464 MEXU) & $\mathrm{T}$ & $1,500,2,000$ & BMM & 6 & \\
\hline $\begin{array}{l}\text { Pteridium caudatum (L.) Maxon (CICH } 486 \text { MEXU) } \\
\text { DICKSONIACEAE }\end{array}$ & $\mathrm{T}$ & 1,000 & ET & 1 & \\
\hline $\begin{array}{l}\text { Lophosoria quadripinnata (J.F.Gmel.) C.Chr. (CICH } 363 \text { MEXU, CIB; } \\
\text { CICH } 413 \text { MEXU; CICH } 634 \mathrm{CIB})\end{array}$ & $\mathrm{T}$ & $\begin{array}{l}1,500,2,000 \\
2,500\end{array}$ & BMM, BPQ & 33 & \\
\hline \multicolumn{6}{|l|}{ DRYOPTERIDACEAE } \\
\hline $\begin{array}{l}\text { Arachniodes denticulata (Sw.) Ching (CICH } 345 \text { MEXU, UC CIB; } \\
\text { CICH } 605 \text { MEXU, CIB) }\end{array}$ & $\mathrm{T}$ & $2,000,2,500$ & BMM, BPQ & 32 & \\
\hline Bolbitis portoricensis (Spreng.) Hennipman (CICH 552 MEXU) & $\mathrm{T}$ & 500 & SMS & 1 & \\
\hline $\begin{array}{l}\text { Megalastrum pulverulentum (Poir.) A.R.Sm. \& R.C.Moran } \\
\quad(\mathrm{CICH} 425 \text { UC, CIB) }\end{array}$ & $\mathrm{T}$ & 1,500 & BMM & 5 & \\
\hline Ctenitis hemsleyana (Baker) Copel. (CICH 606 MEXU, CIB) & $\mathrm{T}$ & 2,000 & $\mathrm{BMM}$ & 10 & \\
\hline Ctenitis (C.Chr.) C.Chr. sp. (CICH 314 MEXU) & $\mathrm{T}$ & 1,500 & BMM & 1 & \\
\hline Dryopteris wallichiana (Spreng.) Hyl. (CICH 340 MEXU, UC, CIB) & $\mathrm{T}$ & $\begin{array}{l}2,000,2,500 \\
3,000\end{array}$ & $\begin{array}{l}\text { BMM, BPQ, } \\
\text { BP }\end{array}$ & 30 & \\
\hline Elaphoglossum erinaceum (Fée) T.Moore (CICH 640 CIB) & $\mathrm{R}$ & 2,000 & BMM & 2 & \\
\hline Elaphoglossum glaucum T.Moore (CICH 386 MEXU, UC, CIB) & $\mathrm{E}$ & 2,500 & BPQ & 4 & \\
\hline Elaphoglossum lonchophyllum (Fée) T.Moore (CICH 431 MEXU, UC) & $\mathrm{E}$ & 1,500 & BMM & 3 & \\
\hline Elaphoglossum muscosum (Sw.) T.Moore (CICH 678 CIB) & $\mathrm{E}$ & 2,000 & $\mathrm{BMM}$ & 2 & \\
\hline Elaphoglossum pallidum C. Chr. (CICH 387 MEXU, UC; CICH 673 CIB) & $\mathrm{E}$ & $2,000,2,500$ & $\mathrm{BMM}, \mathrm{BPQ}$ & 3 & NR \\
\hline Elaphoglossum peltatum (Sw.) Urb. (CICH 422, MEXU, UC, CIB) & $\mathrm{E}$ & $1,500,2,000$ & $\mathrm{BMM}$ & 11 & \\
\hline Elaphoglossum pringlei (Davenp.) C.Chr. (CICH 675 MEXU, CIB) & $\mathrm{E}$ & 2,000 & BMM & 2 & \\
\hline Elaphoglossum sartorii (Liebm.) Mickel (CICH 389 MEXU, UC) & $\mathrm{E}$ & $\begin{array}{l}1,500,2,000 \\
2,500\end{array}$ & $\mathrm{BMM}, \mathrm{BPQ}$ & 13 & \\
\hline Elaphoglossum squamipes (Hook.) T.Moore (CICH 352 MEXU, UC, CIB) & $\mathrm{E}$ & 2,500 & $\mathrm{BPQ}$ & 2 & \\
\hline $\begin{array}{l}\text { Elaphoglossum squarrosum (Klotzsch) T.Moore (CICH } 385 \\
\text { MEXU, UC, CIB) }\end{array}$ & $\mathrm{R}$ & 2,500 & $\mathrm{BPQ}$ & 2 & NR \\
\hline $\begin{array}{l}\text { Elaphoglossum vestitum (Schltdl. \& Cham.) T.Moore }(\mathrm{CICH} \\
\quad 428 \mathrm{MEXU}, \mathrm{UC})\end{array}$ & $\mathrm{E}$ & 1,500 & $\mathrm{BMM}$ & 16 & EM \\
\hline Phanerophlebia macrosora (Baker) Underw. (CICH 348 MEXU, UC, CIB) & $\mathrm{T}$ & 2,000 & BMM & 5 & \\
\hline Phanerophlebia C. Presl. sp. (CICH 801 CIB) & $\mathrm{T}$ & 2,500 & BPQ & 1 & \\
\hline
\end{tabular}


Apéndice 1. Continuación

\begin{tabular}{|c|c|c|c|c|c|}
\hline Familia/Especie (ejemplar de referencia, herbario) & Hábito & $\begin{array}{l}\text { Elevación } \\
\text { (m s.n.m.) }\end{array}$ & Vegetación & No. & $\begin{array}{l}\text { Observa- } \\
\text { ciones }\end{array}$ \\
\hline Polystichum distans E.Fourn. (CICH 332 MEXU, UC) & $\mathrm{T}$ & 2,500 & $\mathrm{BPQ}$ & 3 & \\
\hline $\begin{array}{l}\text { Polystichum hartwegii (Klotzsch) Hieron. (CICH } 344 \text { MEXU, UC; } \\
\text { CICH } 442 \text { MEXU, UC) }\end{array}$ & $\mathrm{T}$ & $1,500,2,500$ & BMM, BPQ & 31 & \\
\hline Polystichum muricatum (L.) Fée (CICH 344 MEXU, UC) & $\mathrm{T}$ & 2,500 & $\mathrm{BPQ}$ & 3 & NR \\
\hline $\begin{array}{l}\text { Polystichum speciosissimum (A.Braun ex Kunze) Copel. } \\
\text { (CICH } 392 \text { MEXU, CIB) }\end{array}$ & $\mathrm{T}$ & $3,000,3,500$ & $\mathrm{BP}, \mathrm{BA}$ & 5 & \\
\hline $\begin{array}{l}\text { Polystichum Roth sp. (CICH } 631 \mathrm{CIB}) \\
\text { GLEICHENIACEAE }\end{array}$ & $\mathrm{T}$ & 2,000 & BMM & 1 & \\
\hline $\begin{array}{l}\text { Sticherus palmatus (W.Schaffn. ex E.Fourn.) Copel. (CICH } 429 \text { MEXU, } \\
\text { CIB; CICH } 752 \text { CIB) }\end{array}$ & $\mathrm{T}$ & $1,500,2,000$ & BMM & 10 & \\
\hline HYMENOPHYLLACEAE & & & & & \\
\hline $\begin{array}{l}\text { Didymoglossum reptans (Sw.) C.Presl (CICH } 484 \text { MEXU, UC; } \\
\text { CICH } 658 \text { MEXU, CIB) }\end{array}$ & $\mathrm{E}$ & $1,500,2,000$ & $\mathrm{BMM}$ & 13 & \\
\hline Hymenophyllum crispum Kunth (CICH $735 \mathrm{CIB})$ & $\mathrm{E}$ & 2,000 & BMM & 1 & \\
\hline $\begin{array}{l}\text { Hymenophyllum fucoides (Sw.) Sw. (CICH } 376 \text { MEXU, UC; } \\
\text { CICH } 646 \mathrm{MEXU}, \mathrm{CIB})\end{array}$ & $\mathrm{E}$ & $2,000,2,500$ & $\mathrm{BMM}, \mathrm{BPQ}$ & 18 & \\
\hline Hymenophyllum polyanthos Bosch (CICH 650 CIB) & $\mathrm{E}$ & 2,000 & BMM & 2 & \\
\hline $\begin{array}{l}\text { Hymenophyllum tegularis (Desv.) Proctor \& Lourteig (CICH } 359 \\
\text { MEXU, UC; } \mathrm{CICH} 660 \text { MEXU) }\end{array}$ & $E$ & $2,000,2,500$ & $\mathrm{BMM}, \mathrm{BPQ}$ & 8 & \\
\hline Hymenophyllum trapezoidale Liebm. (CICH 403 MEXU, UC) & $\mathrm{E}$ & 2,500 & $\mathrm{BPQ}$ & 5 & \\
\hline Hymenophyllum tunbrigense (L.) Sm. (CICH 343 MEXU, UC) & $\mathrm{E}$ & 2,500 & BPQ & 2 & \\
\hline $\begin{array}{l}\text { Polyphlebium capillaceum (L.) Ebihara \& Dubuisson (CICH } 432 \\
\text { MEXU, UC; } \mathrm{CICH} 623 \text { MEXU) }\end{array}$ & $\mathrm{E}$ & $1,500,2,000$ & BMM & 16 & \\
\hline $\begin{array}{l}\text { Polyphlebium hymenophylloides (Bosch) Ebihara \& Dubuisson } \\
\text { (CICH } 662 \mathrm{CIB} ; \mathrm{CICH} 663 \mathrm{CIB})\end{array}$ & $\mathrm{E}$ & 2,000 & BMM & 4 & \\
\hline Polyphlebium pyxidiferum (L.) Ebihara \& Dubuisson (CICH 453 MEXU) & $\mathrm{E}$ & 1,500 & BMM & 4 & \\
\hline $\begin{array}{l}\text { Vandenboschia radicans (Sw.) Copel. (CICH } 405 \text { MEXU, UC; } \\
\text { CICH } 657 \text { MEXU) }\end{array}$ & $\mathrm{E}$ & $2,000,2,500$ & $\mathrm{BMM}, \mathrm{BPQ}$ & 4 & \\
\hline LINDSAEACEAE & & & & & \\
\hline $\begin{array}{l}\text { Lonchitis hirsuta L. (CICH } 520 \text { MEXU, CIB) } \\
\text { LYGODIACEAE }\end{array}$ & $\mathrm{T}$ & 500 & SMS & 1 & \\
\hline $\begin{array}{l}\text { Lygodium venustum Sw. }(\mathrm{CICH} 701 \mathrm{CIB}) \\
\text { MARATTIACEAE }\end{array}$ & ES & 20 & SMS & 9 & \\
\hline $\begin{array}{l}\text { Marattia laxa Kunze (CICH } 412 \text { MEXU, UC; CICH } 635 \text { CIB) } \\
\text { OPHIOGLOSSACEAE }\end{array}$ & $\mathrm{T}$ & $1,500,2,000$ & BMM & 25 & $\operatorname{Pr}$ \\
\hline Botrychium decompositum M.Martens \& Galeotti (CICH 458 MEXU, UC) & $\mathrm{T}$ & 1,500 & BMM & 1 & \\
\hline $\begin{array}{l}\text { Botrychium virginianum (L.) Sw. (CICH } 451 \text { MEXU, UC; CICH } 628 \text { CIB) } \\
\text { PLAGIOGYRIACEAE }\end{array}$ & $\mathrm{T}$ & $1,500,2,000$ & BMM & 2 & \\
\hline $\begin{array}{l}\text { Plagiogyria pectinata (Liebm.) Lellinger (CICH } 333 \text { MEXU, UC) } \\
\text { POLYPODIACEAE }\end{array}$ & $\mathrm{T}$ & 2,500 & $\mathrm{BPQ}$ & 18 & \\
\hline $\begin{array}{l}\text { Alansmia cultrata (Willd.) Moguel \& M.Kessler (CICH } 326 \text { XAL, } \\
\text { CICH } 951 \mathrm{CIB})\end{array}$ & $\mathrm{E}$ & $1,500,2,000$ & BMM & 2 & \\
\hline Campyloneurum amphostenon Fée (CICH 346 MEXU, UC) & $\mathrm{E}$ & $2,000,2,500$ & $\mathrm{BMM}, \mathrm{BPQ}$ & 17 & \\
\hline $\begin{array}{l}\text { Campyloneurum angustifolium Fée (CICH } 368 \text { MEXU, UC; } \\
\text { CICH } 454 \text { MEXU, UC) }\end{array}$ & $\mathrm{E}$ & $1,500,2,500$ & BMM, BP & 10 & \\
\hline Campyloneurum phyllitidis C.Presl (CICH 733 CIB; CICH 518 MEXU) & $\mathrm{E}$ & $500,1,000$ & SMS, ET & 14 & A \\
\hline Cochlidium linearifolium (Desv.) Maxon ex C.Chr. (CICH 371 MEXU, CIB) & $\mathrm{E}$ & 2,500 & $\mathrm{BPQ}$ & 3 & \\
\hline $\begin{array}{l}\text { Melpomene leptostoma (Fée) A.R.Sm. \& R.C.Moran (CICH } \\
328 \text { MEXU, UC) }\end{array}$ & $E$ & 1,500 & $\mathrm{BMM}$ & 1 & \\
\hline
\end{tabular}


Apéndice 1. Continuación

\begin{tabular}{|c|c|c|c|c|c|}
\hline Familia/Especie (ejemplar de referencia, herbario) & Hábito & $\begin{array}{l}\text { Elevación } \\
\text { (m s.n.m.) }\end{array}$ & Vegetación & No. & $\begin{array}{l}\text { Observa- } \\
\text { ciones }\end{array}$ \\
\hline $\begin{array}{l}\text { Melpomene pilosissima (M.Martens \& Galeotti) A.R.Sm. \& R.C.Moran } \\
(\mathrm{CICH} 329 \mathrm{MEXU}, \mathrm{UC} \text {; } \mathrm{CICH} 372 \mathrm{MEXU}, \mathrm{UC})\end{array}$ & $\mathrm{E}$ & $1,500,2,500$ & $\mathrm{BMM}, \mathrm{BPQ}$ & 9 & \\
\hline $\begin{array}{l}\text { Melpomene xiphopteroides (Liebm.) A.R.Sm. \& R.C.Moran } \\
\text { (CICH } 665 \text { MEXU) }\end{array}$ & $\mathrm{E}$ & $1,500,2,000$ & BMM & 5 & \\
\hline Microgramma nitida (J.Sm.) A.R.Sm. (CICH 487 MEXU, UC) & $\mathrm{E}$ & $\begin{array}{l}20,500 \\
1,000\end{array}$ & SMS, ET & 9 & \\
\hline $\begin{array}{l}\text { Pecluma atra (A.M.Evans) M.G.Price (CICH } 532 \text { CIB; } \\
\text { CICH } 498 \text { MEXU, UC) }\end{array}$ & $\mathrm{E}$ & $500,1,000$ & SMS, ET & 6 & \\
\hline Pecluma divaricata (E.Fourn.) Mickel \& Beitel (CICH 489 CIB) & $\mathrm{E}$ & 1,000 & ET & 8 & \\
\hline Pecluma sursumcurrens (Copel.) M.G.Price (CICH 435 MEXU, UC) & $\mathrm{E}$ & 1,500 & BMM & 18 & \\
\hline Phlebodium pseudoaureum (Cav.) Lellinger (CICH 419 MEXU) & $\mathrm{E}$ & $\begin{array}{l}1,000,1,500 \\
2,000\end{array}$ & $\mathrm{ET}, \mathrm{BMM}$ & 37 & \\
\hline Pleopeltis angusta var. stenoloma (Fée) Farw. (CICH 416 MEXU) & $\mathrm{E}$ & 1,500 & $\mathrm{BMM}$ & 22 & \\
\hline Pleopeltis crassinervata (Fée) T.Moore (CICH 423 MEXU, UC) & $\mathrm{E}$ & 1,500 & BMM & 17 & \\
\hline $\begin{array}{l}\text { Pleopeltis furfuracea (Schltdl. \& Cham.) A.R.Sm. \& Tejero } \\
\text { (CICH } 457 \text { MEXU) }\end{array}$ & $\mathrm{E}$ & $\begin{array}{l}500,1,000 \\
1,500\end{array}$ & $\begin{array}{l}\text { SMS, ET, } \\
\text { BMM }\end{array}$ & 22 & \\
\hline Pleopeltis lepidotricha (Fée) A.R.Sm. \& Tejero (CICH 456 MEXU, UC) & $\mathrm{E}$ & 1,500 & BMM & 5 & \\
\hline Pleopeltis mexicana (Fée) Mickel \& Beitel (CICH 318 MEXU, UC) & $\mathrm{E}$ & $1,500,2,000$ & $\mathrm{BMM}$ & 5 & \\
\hline Pleopeltis plebeia (Schltdl. \& Cham.) A.R.Sm. \& Tejero (CICH 338 MEXU) & $\mathrm{E}$ & $\begin{array}{l}1,500,2,000 \\
2,500,3,000 \\
3,500\end{array}$ & $\begin{array}{l}\mathrm{BMM}, \mathrm{BPQ}, \\
\mathrm{BP}, \mathrm{BA}\end{array}$ & 68 & \\
\hline $\begin{array}{l}\text { Pleopeltis polylepis var. interjecta (Weath.) E.A.Hooper } \\
\text { (CICH } 648 \mathrm{MEXU})\end{array}$ & $\mathrm{E}$ & $\begin{array}{l}2,000,2,500 \\
3,500\end{array}$ & $\begin{array}{l}\mathrm{BMM}, \mathrm{BPQ}, \\
\mathrm{BA}\end{array}$ & 24 & \\
\hline $\begin{array}{l}\text { Pleopeltis polylepis var. polylepis (Roem. ex Kunze) T. Moore } \\
\quad(\mathrm{CICH} 395 \mathrm{MEXU}, \mathrm{UC})\end{array}$ & $\mathrm{E}$ & 3,000 & $\mathrm{BP}$ & 15 & \\
\hline $\begin{array}{l}\text { Pleopeltis polypodioides (L.) E.G.Andrews \& Windham } \\
\quad(\mathrm{CICH} 681 \mathrm{MEXU}, \mathrm{UC})\end{array}$ & $\mathrm{E}$ & $20,2,000$ & SMS, BMM & 8 & \\
\hline Polypodium californicum Kaulf. (CICH 719 CIB) & $\mathrm{R}$ & 3,500 & BA & 1 & \\
\hline Polypodium conterminans E.Fourn. (CICH 653 MEXU) & $\mathrm{E}$ & 2,000 & $\mathrm{BMM}$ & 17 & \\
\hline Polypodium hartwegianum Hook. (CICH 370 MEXU, UC) & $\mathrm{E}$ & 3,000 & $\mathrm{BPQ}, \mathrm{BP}$ & 3 & \\
\hline Polypodium longepinnulatum E.Fourn. (CICH 322 MEXU, UC) & $\mathrm{E}$ & $1,500,2,000$ & $\mathrm{BMM}$ & 3 & \\
\hline Polypodium martensii Mett. (CICH 397 MEXU, UC) & $\mathrm{E}$ & 2,500 & BPQ & 19 & \\
\hline Polypodium plesiosorum Kunze (CICH 342 MEXU) & $\mathrm{E}$ & 2,500 & $\mathrm{BPQ}$ & 14 & \\
\hline Polypodium puberulum Schltdl. \& Cham. (CICH 463 MEXU) & $\mathrm{E}$ & $\begin{array}{l}1,500,2,000 \\
2,500\end{array}$ & $\mathrm{BMM}, \mathrm{BPQ}$ & 17 & \\
\hline Polypodium rhodopleuron Kunze (CICH 437 MEXU) & $\mathrm{E}$ & 1,500 & BMM & 4 & \\
\hline Serpocaulon falcaria (Kunze) A.R.Sm. (CICH 426 MEXU, UC) & $\mathrm{E}$ & $1,500,2,000$ & BMM & 22 & \\
\hline Serpocaulon triseriale (Sw.) A.R.Sm. (CICH 493 MEXU, UC) & $\mathrm{E}$ & $500,1,000$ & SMS, ET & 8 & A \\
\hline $\begin{array}{l}\text { Terpsichore asplenifolia (L.) A.R.Sm. (CICH } 448 \text { MEXU, UC) } \\
\text { PSILOTACEAE }\end{array}$ & $\mathrm{E}$ & 1,500 & BMM & 6 & \\
\hline $\begin{array}{l}\text { Psilotum complanatum Sw. (CICH } 415 \text { MEXU, UC) } \\
\text { PTERIDACEAE }\end{array}$ & $\mathrm{E}$ & $1,500,2,000$ & BMM & 13 & A \\
\hline Adiantum andicola Liebm. (CICH 455 MEXU; CICH 527 MEXU) & $\mathrm{T}$ & $500,1,500$ & $\mathrm{ET}, \mathrm{BMM}$ & 18 & \\
\hline Adiantum capillus-veneris L. (CICH 522 CIB, 669 MEXU) & $\mathrm{T}$ & $500,2,000$ & $\mathrm{ET}, \mathrm{BMM}$ & 9 & \\
\hline Adiantum concinnum Humb. \& Bonpl. ex Willd. (CICH 537 MEXU) & Ty $R$ & 500 & ET & 2 & \\
\hline Adiantum poiretii Wikstr. (CICH 313 MEXU) & $\mathrm{T}$ & 1,500 & BMM & 1 & \\
\hline Adiantum tenerum (CICH 491 MEXU, UC) & $\mathrm{T}$ & 1,000 & ET & 9 & \\
\hline Anogramma leptophylla (L.) Link (CICH 500 MEXU) & $\mathrm{R}$ & 500 & SMS & 3 & \\
\hline Astrolepis sinuata (Lag. ex Sw.) D.M.Benham \& Windham (CICH 516 CIB) & $\mathrm{R}$ & 3,000 & $\mathrm{BP}$ & 1 & \\
\hline
\end{tabular}


Apéndice 1. Continuación

Familia/Especie (ejemplar de referencia, herbario)

Gaga marginata (Kunth) F.W.Li \& Windham (CICH 384 MEXU, UC)

Hemionitis palmata L. (CICH $490 \mathrm{MEXU})$

Myriopteris lendigera (Cav.) J.Sm. (CICH 393 MEXU, UC)

Myriopteris microphylla (Sw.) Grusz \& Windham (CICH 499 MEXU, UC)

Pityrogramma dealbata (C.Presl) Domin (CICH 534 MEXU)

Polytaenium lineatum (Sw.) J.Sm. (CICH 446 MEXU, UC)

Pteris longifolia L. (CICH $539 \mathrm{CIB})$

Pteris muricata Hook. (CICH 479 MEXU; CICH 612 MEXU)

Pteris orizabae M.Martens \& Galeotti (CICH 383 MEXU, UC)

Pteris podophylla Sw. (CICH $445 \mathrm{MEXU})$

Scoliosorus ensiformis (Hook.) T.Moore (CICH 331 MEXU, UC)

Vittaria graminifolia Kaulf. (CICH 320 MEXU; CICH 337 MEXU)

Vittaria Sm sp. (CICH $802 \mathrm{CIB})$

SACCOLOMATACEAE

Saccoloma inaequale (Kunze) Mett. (CICH 316 MEXU)

TECTARIACEAE

Tectaria heracleifolia (Willd.) Underw. (CICH 494 MEXU,

UC; CICH 519 MEXU)

\section{THELYPTERIDACEAE}

Macrothelypteris torresiana (Gaudich.) Ching (CICH $521 \mathrm{MEXU})$

Thelypteris dentata (Forssk.) E.P.St.John (CICH 462 MEXU, UC)

Thelypteris kunthii (Desv.) C.V.Morton (CICH 524 MEXU)

Thelypteris melanochlaena (C.Chr.) C.F.Reed ( $\mathrm{CICH} 461 \mathrm{MEXU}, \mathrm{UC})$

Thelypteris rudis (Kunze) Proctor (CICH 315 MEXU; CICH 360 MEXU)

Thelypteris Adans sp. (CICH $700 \mathrm{CIB})$

WOODSIACEAE

Athyrium arcuatum Liebm. (CICH $380 \mathrm{MEXU}, \mathrm{UC})$

Cystopteris fragilis (L.) Bernh. (CICH 377 CIB; CICH 394 MEXU)

Diplazium donnell-smithii Christ ( $\mathrm{CICH} 441 \mathrm{MEXU}, \mathrm{UC})$

Diplazium franconis Liebm. (CICH 436 MEXU, UC; CICH 523 MEXU)

Diplazium striatum (L.) C.Presl (CICH 685 MEXU)

Diplazium ternatum Liebm. (CICH 323 MEXU, UC;

CICH 639 MEXU, UC)

\begin{tabular}{|c|c|c|c|c|}
\hline Hábito & $\begin{array}{l}\text { Elevación } \\
\text { (m s.n.m.) }\end{array}$ & Vegetación & No. & $\begin{array}{c}\text { Observa- } \\
\text { ciones }\end{array}$ \\
\hline $\mathrm{T}$ & 2,500 & $\mathrm{BPQ}$ & 2 & \\
\hline $\mathrm{T}$ & 1,000 & ET & 1 & \\
\hline $\mathrm{T}$ & 3,000 & $\mathrm{BP}$ & 1 & \\
\hline $\mathrm{T}$ & $500,1,000$ & SMS, ET & 11 & \\
\hline $\mathrm{T}$ & 500 & SMS & 1 & \\
\hline $\mathrm{E}$ & 1,500 & BMM & 2 & \\
\hline $\mathrm{T}$ & 500 & SMS & 1 & \\
\hline $\mathrm{T}$ & $1,500,2,000$ & $\mathrm{BMM}$ & 7 & \\
\hline $\mathrm{T}$ & $1,500,2,500$ & $\mathrm{BMM}, \mathrm{BPQ}$ & 13 & \\
\hline $\mathrm{T}$ & 1,500 & $\mathrm{BMM}$ & 4 & \\
\hline$E$ & $2,000,2,500$ & $\mathrm{BMM}, \mathrm{BPQ}$ & 13 & \\
\hline $\mathrm{E}$ & $\begin{array}{l}1,500,2,000 \\
2,500\end{array}$ & $\mathrm{BMM}, \mathrm{BPQ}$ & 29 & \\
\hline $\mathrm{E}$ & 1,000 & ET & 1 & \\
\hline $\mathrm{T}$ & 1,500 & BMM & 5 & \\
\hline $\mathrm{T}$ & $500,1,000$ & SMS, ET & 15 & \\
\hline $\mathrm{T}$ & 500 & SMS & 4 & \\
\hline $\mathrm{T}$ & 1,500 & BMM & 4 & \\
\hline $\mathrm{T}$ & 500 & SMS & 5 & \\
\hline $\mathrm{T}$ & $500,1,500$ & SMS, BMM & 10 & \\
\hline $\mathrm{T}$ & $\begin{array}{l}1,500,2,000 \\
2,500\end{array}$ & $\mathrm{BMM}, \mathrm{BPQ}$ & 8 & \\
\hline $\mathrm{T}$ & 20 & SMS & 5 & \\
\hline $\mathrm{T}$ & 2,500 & BMM & 9 & \\
\hline $\mathrm{T}$ & $2,500,3,000$ & $B P Q, B P$ & 15 & \\
\hline $\mathrm{T}$ & 1,500 & BMM & 2 & \\
\hline $\mathrm{T}$ & $\begin{array}{l}500,1,500, \\
2,000\end{array}$ & SMS, BMM & 16 & \\
\hline $\mathrm{T}$ & 2,000 & BMM & 4 & \\
\hline $\mathrm{T}$ & $2,000,2,500$ & $\mathrm{BMM}, \mathrm{BPQ}$ & 12 & \\
\hline
\end{tabular}

